\title{
Modelo de avaliação da viabilidade econômico- financeira da implantação de usinas de reciclagem de resíduos da construção civil em municípios brasileiros
}

\author{
Economic and financial viability evaluation model for the implementation \\ of waste recycling plants construction in Brazilian municipalities
}

\section{Luis Carlos Pais Gularte"* ${ }^{\circledR}$, José Donizetti de Lima' ${ }^{\oplus}$, Gilson Adamczuk Oliveira' ${ }^{\circledR}$, Rodrigo Barichello ${ }^{2}$, Michele Aparecida Nepomuceno Pinto $^{3}$ ()}

口-

\begin{abstract}
RESUMO
Este trabalho teve como objetivo geral desenvolver um modelo de avaliação da viabilidade econômico-financeira da implantação de usinas de reciclagem de resíduos da construção civil e demolição (URRCD) em municípios brasileiros. O modelo de avaliação proposto para cada projeto de investimento (PI) em estudo uniu o modelo de negócio Canvas, o plano de negócios, a metodologia multi-índice proposta por Souza e Clemente (2009) e ampliada por Lima et al. (2015) por meio do aplicativo web \$AVEPI. Também foi realizada uma análise de sensibilidade por meio de limites de elasticidade e valores-limite, bem como uma análise estocástica utilizando a simulação de Monte Carlo (SMC). Com base na proposição desse modelo, este foi aplicado em um estudo de caso no município de Pato Branco (PR). Os resultados mostram que há viabilidade econômicofinanceira na implantação desse PI, uma vez que o valor presente líquido médio é de aproximadamente $\mathrm{R} \$ 583.919,87$, e a recuperação do investimento se concretizará em 12 anos (payback). Por meio da SMC, visualizou-se que há alta probabilidade (99,78\%) de que o investimento na URRCD de Pato Branco seja rentável. Entretanto, os índices de elasticidade mostraram que é preciso monitorar as receitas esperadas e os custos estimados com mais atenção. Assim, será preciso um trabalho, por parte dos gestores da URRCD, em prol de sempre estarem buscando aumentar a demanda pelos agregados reciclados, como maneira de aumentar a receita da usina, desde que se respeite a sua capacidade de produção. Palavras-chave: usina de reciclagem de resíduos da construção civil; viabilidade econômico-financeira; modelo de negócio canvas; metodologia multi-índice ampliada; simulação de Monte Carlo; \$AVEPI.
\end{abstract}

\begin{abstract}
This work had as main objective to develop an evaluation model for analyzing the economic and financial feasibility of implementing Construction and Demolition Waste Recycling Plants (URRCD) in Brazilian municipalities. The evaluation model proposed for the Investment Project (IP) under study was performed using the business model Canvas, the business plan, the multiindex method proposed by Souza and Clemente (2009) and expanded by Lima et al. (2015) through the \$AVEPI Web app. Also, a sensitivity analysis was performed, through the elasticity limits and limit values as well as a stochastic analysis using the Monte Carlo Simulation (MCS). From the proposition of this model, it was applied in a case study in Pato Branco, Paraná. The results showed that there is economic and financial viability in the implementation of this IP, since the average Net Present Value (NPV) is about R\$ 583,919.87 and the investment recovery will materialize in 12 years (Payback). Through MCS, it was observed that there is a high probability (99.78\%) that the investment in the URRCD of Pato Branco, Paraná, be profitable. However, the elasticity indices showed that it is necessary to monitor the expected revenues and the estimated costs with more attention. Thus, it will require a work by the managers of the URRCD, in favor of always seeking to increase the demand for recycled aggregates, as a way to increase the revenue of the plant, as long as it does not exceed its production capacity.
\end{abstract}

Keywords: construction waste recycling plant; economic and financial viability; business model canvas; extended multi-index methodology; Monte Carlo simulation; \$AVEPI.

\section{INTRODUÇÃO}

Com o desenvolvimento das cidades e o rápido crescimento da população, um problema que não pode ser mais evitado é o do destino e uso correto dos resíduos. Entre os resíduos sólidos urbanos gerados nas cidades brasileiras, os resíduos da construção civil e demolição (RCD) representam aproximadamente $60 \%$. Esses resíduos são oriundos de

'Universidade Tecnológica Federal do Paraná - Pato Branco (PR), Brasil.

¿Universidade Comunitária da Região de Chapecó - Chapecó (SC), Brasil.

${ }^{3}$ Universidade Estadual de Maringá - Maringá (PR), Brasil.

*Autor correspondente: luisgularte755@hotmail.com

Recebido: 20/03/2018 - Aceito: 05/02/2019 - Reg. Abes: 193153 
demolições, reformas e de novas construções e quando são depositados de forma inadequada geram problemas à sociedade e ao meio ambiente (ABRELPE, 2016).

Com a publicação da Resolução do Conselho Nacional do Meio Ambiente (Conama) no 307/02 (BRASIL, 2002) e da Norma Técnica Brasileira (NBR) 15.114/04, da Associação Brasileira de Normas Técnicas (ABNT), foram definidas diretrizes acerca do gerenciamento dos RCD. Por meio dessas normatizações, a responsabilidade pela gestão dos RCD passou da administração pública para os geradores. Considerando que essa resolução preconiza a não geração de resíduos como o elemento básico para a gestão dos canteiros de obras, colocou-se, a partir daí, uma nova realidade para a construção civil no Brasil.

Contudo, atualmente a maioria dos RCD ainda é enviada para aterros, reutilizada ou incinerada, de maneira precária. De acordo com a Associação Brasileira de Empresas de Limpeza Pública e Resíduos Especiais (ABRELPE, 2016), em torno de 40\% de todo o resíduo gerado pelo setor é despejado em aterros. Desse montante, $53 \%$ é reutilizado ou incinerado, o que mostra que apenas $5 \%$ é reciclado. A quantidade de resíduos reciclados no Brasil é baixa se comparada com outros países como os Estados Unidos, no qual se reciclam 140 milhões de toneladas por ano (ABRECON, 2015). Isso é um reflexo das poucas usinas de reciclagem de resíduos da construção civil e demolição (URRCD) que atualmente estão instaladas no Brasil (apenas 48), segundo dados da Associação Brasileira para Reciclagem de Resíduos da Construção e Demolição (ABRECON, 2015). No Paraná há apenas sete em funcionamento.

De acordo com dados da Abrelpe (2016), a geração estimada de RCD no Brasil seria de 122.262 toneladas por dia. Esses dados mostram o elevado potencial que o setor tem em produzir agregados reciclados, os quais podem agregar valor à produção. Com isso, há diminuição da necessidade de matérias-primas virgens, provocando redução nos custos das empresas e causando impacto socioambiental positivo, uma vez que esses resíduos deixam de ser descartados em aterros ou até no próprio meio ambiente (PINTO, 1999).

Por meio da publicação da Resolução Conama no 307/02 (BRASIL, 2002) e da NBR 15.114, da ABNT, em 2004, colocou-se uma nova realidade para a construção civil no Brasil. Assim, a logística reversa no setor tornou-se uma obrigação para as empresas que prezam a gestão da qualidade. Contudo, ainda hoje são poucos os casos de implantação efetiva de URRCD no país (ABRELPE, 2016). Isso se deve, em parte, à falta de planos de manejo e gestão por parte dos municípios brasileiros que incentivem a redução, orientem o manejo ou facilitem a adequada destinação dos resíduos do setor. Porém, parte indispensável de qualquer plano de manejo e gestão de RCD é a implantação de uma usina para reciclar esses resíduos, seja ela pública e/ou privada, atendendo a um ou mais municípios. Entende-se que a implantação dessa usina não pode ocorrer sem a prévia realização de um planejamento apropriado por meio de um plano de negócio e com a avaliação de sua viabilidade econômico-financeira. A avaliação da viabilidade econômico-financeira de implantação de uma URRCD foi o escopo deste estudo.

Quanto à contribuição prática da pesquisa, entende-se que este trabalho: permite aos gestores promoverem ações de destinação adequadas, atendendo às normatizações ambientais, e ainda possibilitará aumento na competitividade do setor. Além disso, um estudo de viabilidade econômica mostra-se um importante suporte à tomada de decisão dos gestores das indústrias do setor de construção civil e de órgãos públicos. A contribuição teórica da pesquisa ocorre no campo da proposição de um modelo que une o modelo canvas com o plano de negócios e a metodologia multi-índice ampliada (MMIA) com o uso da simulação de Monte Carlo (SMC) como instrumento de avaliação da viabilidade econômico-financeira da implantação de usinas de RCD em municípios brasileiros.

Com o uso desse modelo, o qual possui mais de 50 indicadores, além da abordagem probabilística por meio da SMC e do plano de negócios completo, esta pesquisa apresentou uma análise detalhada e específica do objeto de estudo, permitindo um parecer mais específico a respeito da viabilidade ou não de projetos de usinas em municípios brasileiros. Como se trata de um modelo aplicável a municípios brasileiros, entende-se que este trabalho poderá servir como base a possíveis investidores e gestores que tenham como objetivo implantar uma usina de RCD em seus municípios, e, como forma de testar empiricamente o modelo proposto, foi feito um estudo de caso na cidade de Pato Branco (PR).

$\mathrm{O}$ artigo está dividido da seguinte forma: a revisão de literatura, a metodologia aplicada neste trabalho, o modelo de avaliação da viabilidade econômico-financeira e a sua aplicação em um estudo de caso. Por fim, são feitas as considerações finais.

\section{REVISÃO DE LITERATURA}

\section{Resíduos da construção civil}

A reciclagem de RCD é uma prática que apresenta vantagens ambientais à sociedade, tais como: economia na aquisição de matérias-primas, decréscimo da poluição gerada pelo entulho e de suas consequências, como enchentes e assoreamento de rios e córregos, preservação e redução das áreas de aterros inertes (PINTO, 1999). Porém, conforme destacam Santos e Pompeu (2014), para o meio empresarial, o argumento referindo-se somente a questões ambientais ou sustentáveis se torna insuficiente, principalmente na atividade proposta, pois a instalação de uma URRCD demanda investimentos elevados. Assim, torna-se necessário um planejamento econômico-financeiro para avaliar a viabilidade de um empreendimento desse porte. Portanto, é necessário justificar a reciclagem desses resíduos como uma atividade que, entre outros aspectos, gere emprego e renda e que seja economicamente lucrativa. 
Analisando especificamente a questão da avaliação do investimento de um projeto desse porte, há muitos estudos que buscaram analisar a viabilidade econômica de se implantar uma URRCD - como exemplos, tem-se: Duran, Lenihan e O'Regan (2006), Zhao, Leeftink e Rotter (2010), Coelho e Brito (2013), em âmbito internacional, e Pinto (1999) e Gularte et al. (2017), em âmbito nacional.

Entretanto, diferentes resultados têm sido encontrados na literatura: Nunes et al. (2007) não encontraram viabilidade no investimento privado em projetos de novas usinas. Por outro lado, Zhao, Leeftink e Rotter (2010) encontraram viabilidade condicional (colocaram a necessidade da intervenção do setor público como fator necessário à viabilidade ou até sugeriram a própria gestão deste). Já estudos como o de Duran, Lenihan e O’Regan (2006) e Gularte et al. (2017) apresentaram retornos elevados de investimentos.

Zhao, Leeftink e Rotter (2010) buscaram avaliar se a reciclagem dos RCD seria viável na cidade de Chongqing, China. Ao analisar as condições econômicas da região, eles verificaram que havia elevada quantidade de resíduos e expressiva demanda por materiais reciclados por conta das movimentadas atividades de construção em curso, o que gerou elevado potencial de mercado e também trouxe um desafio para o fortalecimento do setor de reciclagem, porém a viabilidade econômica só seria alcançada com a aquisição de equipamento usado e se fosse atingida a economia de escala na produção dos agregados reciclados.

Tratando-se do caso brasileiro, o trabalho de Nunes et al. (2007) mostra-se um dos mais importantes estudos da viabilidade econômica de implantação de URRCD privada no país. Os autores analisaram duas possibilidades de usinas: uma com capacidade de operação de 20 ton/h e outra de 100 ton/h. Analisando casos de usinas já existentes e considerando as condições do mercado brasileiro na época, os autores concluem que os modelos analisados apenas seriam economicamente viáveis se houvesse algum incentivo do poder público, como subvenção de impostos e taxas, ou, dependendo da condição do município, as autoridades públicas poderiam até mesmo assumir a gestão do empreendimento.

Além do exposto, a análise dessas publicações permite depreender que há diversos modelos para aferir-se a viabilidade econômica de uma URRCD. Como este trabalho visou fazer uma análise de investimento em uma usina privada, devem-se determinar, em um primeiro momento, quais indicadores de viabilidade serão considerados na análise. Dessa forma, a segunda parte desta seção apresenta, em síntese, as principais metodologias que são adotadas nos principais estudos de investimentos em uma URRCD.

\section{Análise de viabilidade econômica de projetos de investimento}

Investimento pode ser definido como uma questão de escolha entre alternativas que envolve algum tipo de preferência mediante um custo de oportunidade (HARZER, 2015). O investidor, no momento de decisão, deve considerar todas as opções de investimentos disponíveis no mercado que sejam capazes de gerar retornos mais favoráveis. O objetivo é que, ao final do período de maturidade do investimento, o retorno seja, no mínimo, equivalente ao custo de oportunidade assumido (CASAROTTO FILHO; KOPITTKE, 2010). De acordo com Harzer (2015), quando o investimento destina a aplicação de recursos para a abertura de novos empreendimentos, costuma-se chamá-lo projeto de investimento (PI), sendo esse o adotado.

Um PI constitui-se na elaboração de um modelo, o mais próximo possível do cenário real, ao qual o empreendimento estará sujeito ao longo do período de maturação (HARZER, 2015). O PI abrange a construção de um ou vários cenários imaginários projetados para o futuro negócio, que são elaborados com base nas informações disponíveis no presente e representam as expectativas dos empreendedores acerca do futuro, as quais são manejadas com diversos graus de confiança. Cada cenário contém um conjunto de variáveis e parâmetros de influência coerentes com o modelo, segundo o entendimento de quem decide pelo investimento e representa um esforço para melhorar a qualidade da informação sobre as implicações desejadas ou não, a fim de diminuir os riscos do investimento (SOUZA; CLEMENTE, 2009). Com o intuito de auxiliar na elaboração desse PI, é feito o modelo de negócios Canvas.

O modelo de negócios Canvas surgiu em 2004 e foi desenvolvido pelo pesquisador suíço Alexander Osterwalder. É uma ferramenta de gerenciamento estratégico, a qual permite desenvolver e esboçar modelos de negócio novos ou existentes. Basicamente é um mapa visual pré-formatado contendo nove blocos do modelo de negócios (GAVA, 2014). Esses blocos são apresentados na Figura 1. No modelo de negócios Canvas, cada bloco é como uma organização, pois cria, entrega e captura valor, podendo ser verificado o modo como cada componente contribui para a geração de valor. Essa ferramenta permite identificar, por meio de técnicas de criação do conhecimento, os elementos possíveis e imagináveis que devem compor determinado modelo de negócio.

Com a montagem desses blocos, parte-se para a confecção dos cenários, o que permite identificar pontos fortes e fracos de uma organização, reconhecendo oportunidades e ameaças (análise SWOT,

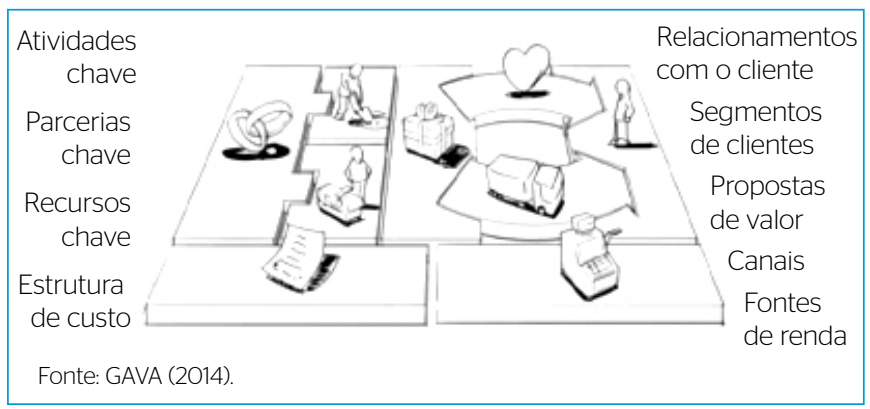

Figura 1 - Representação gráfica das relações entre os componentes de um modelo. 
sigla em inglês para strengths, weaknesses, opportunities and threats). No processo de desenvolvimento de um modelo de negócios, a construção de cenários permite detalhamento do contexto do projeto, especificando o problema. O objetivo não é prever o futuro, mas imaginar possibilidades futuras com detalhe de ideias (GAVA, 2014). Por meio desse modelo de negócios, o PI pode seguir suas etapas convencionais: fixar o horizonte de tempo da variável em estudo, definir as principais variáveis de influência e fixar os parâmetros qualitativos e quantitativos coerentes com as variáveis de influência por meio do modelo de análise de investimento escolhido.

Existem várias abordagens que podem ser utilizadas para se fazer uma análise de investimento. Nogas, Souza e Silva (2011) classificam as abordagens para análise de investimentos em três categorias: metodologia clássica (MC); teoria das opções reais (TOR) e metodologia multi-índice (MMI). Este trabalho utilizará essa última abordagem.

A MMI caracteriza-se pelo uso simultâneo de dois conjuntos de indicadores para representar as dimensões retorno e riscos de um PI, resultando em informações mais consistentes (SOUZA; CLEMENTE, 2009). O primeiro conjunto é representado pelo valor presente líquido (VPL); VPL anualizado (VPLA); índice benefício/custo (IBC); retorno adicional proporcionado pela decisão de investir (ROIA); e índice ROIA/TMA, sendo TMA a taxa mínima de atratividade. O segundo conjunto, representando os riscos, é constituído de índice TMA/TIR (risco financeiro ou proxy de $\mathrm{P}(\mathrm{VPL} \leq 0)$ ), sendo TIR a taxa interna de retorno; índice payback/N (representando a fração da vida útil necessária para a recuperação do capital investido), sendo $\mathrm{N}$ o horizonte de planejamento; grau de comprometimento da receita; risco de negócio $(\mathrm{RN})$; e risco de gestão (RG).

Segundo Harzer (2015), embora a maioria dos indicadores de retorno do PI sejam os mesmos utilizados na MC, a contribuição da MMI reside em sintetizar as informações de retorno e dos riscos, permitindo o confronto dessas dimensões. Na MMI, VPL $>0$ e/ou TIR > TMA apenas indicam que o PI deve continuar sendo analisado. A decisão de recomendar ou não o PI é definida pelo confronto entre o retorno esperado e os riscos percebidos.

Recentemente, Lima et al. (2015) incorporaram à MMI alguns índices para uma análise de sensibilidade (AS) por meio de limites de elasticidade (LEs) e valores-limite (VLs), passando a denominá-la de MMIA. A AS quantifica a sensibilidade dos resultados do PI em relação às mudanças em uma variável, mantendo as demais inalteradas (CORREIA NETO, 2009). Quando uma pequena variação em um parâmetro muda drasticamente a lucratividade de um PI, diz-se que este é muito sensível a esse parâmetro. Logo, é importante concentrar esforços para obter menos dados incertos (LIMA et al., 2015). O propósito principal da AS é aprofundar a percepção de riscos ao qual o PI é submetido (CASAROTTO FILHO; KOPITTKE, 2010; LIMA et al., 2015).
Entretanto, um aspecto que deve ser considerado no processo de tomada de decisão é a incerteza. A incerteza introduz uma nova dimensão na análise de um PI. Isso se deve à impossibilidade de controle de futuros eventos com precisão, tais como comportamento futuro da economia, vendas futuras, preços, custos com desgaste e manutenção de equipamento. Apesar disso, o tomador de decisão precisa definir, sustentado pelas informações disponíveis, qual a melhor decisão a tomar (SOUZA; CLEMENTE, 2009; LIMA et al. 2015).

Segundo Souza e Clemente (2009), na análise de investimentos, deve-se fazer uso de técnicas que levem em conta a aleatoriedade dos elementos que compõem o fluxo de caixa (FC) de um PI. A mais conhecida técnica para lidar com risco e incerteza é a SMC, a qual é uma alternativa adequada para a avaliação do risco de um PI. A SMC é uma técnica que envolve o uso de números pseudoaleatórios e de distribuição de probabilidades para a solução de problemas de diversas áreas de pesquisa (GUJARATI, 2002). A principal vantagem da SMC é determinar quanto uma variação aleatória, que já é conhecida, afeta o desempenho da viabilidade do sistema que está sendo modelado. Com o uso da SMC, os indicadores deixam de ser determinísticos e tornam-se estocásticos. Isso pode favorecer a tomada de decisões de forma mais assertiva (LIMA et al., 2017b).

\section{METODOLOGIA}

O presente trabalho propôs um modelo para avaliação da viabilidade econômica de implantação de URRCD em municípios brasileiros, o qual engloba três partes: elaboração do modelo Canvas com o plano de negócios, aplicação da abordagem determinística via MMIA para analisar a viabilidade econômico-financeira do PI e aplicação da abordagem estocástica por meio da SMC como forma de reduzir a incerteza no PI.

A primeira etapa do trabalho consistiu em construir um referencial teórico com base em pesquisas bibliográfica e documental a respeito dos temas resíduos da construção civil e demolição e viabilidade econômico-financeira de um PI. A segunda etapa, já dentro do modelo proposto, buscou verificar os aspectos mais relevantes da URRCD e na definição dos critérios da empresa, os quais servem de base para a montagem do plano de negócios.

A terceira etapa consistiu no modelo proposto em si, que segue os passos: parte-se do modelo Canvas, o qual deve ser realizado de forma criteriosa pelo empreendedor. Depois, é elaborado o plano de negócios, considerando todos os aspectos relevantes do investimento, como estrutura, marketing, vendas, recursos humanos, entre outros; com essas definições de como funcionarão a empresa, seu tamanho, número de trabalhadores etc., deve ser projetado o FC, que servirá de base para a aplicação das abordagens determinística e estocástica via SMC. A quarta etapa deste trabalho foi a aplicação do modelo proposto por meio do estudo de caso da implantação de uma URRCD no município de Pato Branco. 


\section{Proposição de um modelo de avaliação da viabilidade econômico-financeira para usinas de reciclagem de resíduos da construção civil e demolição}

Para analisar a viabilidade econômico-financeira da implantação de URRCD em municípios brasileiros, este estudo propôs um modelo de avaliação que une o modelo Canvas ao plano de negócios da futura URRCD e a MMIA à SMC. Todas as etapas da aplicação do modelo estão apresentadas na Figura 2. Para implementar o modelo, parte-se da análise do município no qual será instalada a URRCD. Assim, os primeiros dados que devem ser coletados se referem ao número de habitantes da cidade, à projeção de crescimento da população no período analisado e à média da geração de RCD atual (kg/hab/dia). Com base nesses dados, aplica-se o modelo Canvas, no qual o investidor visa estruturar seu negócio por meio da análise global da empresa, seus pontos fortes e fracos, seu mercado consumidor, entre outros.

Após responder a todas essas questões é definido o modelo de negócio do qual se partirá para construir o plano de negócios. Posteriormente, são feitas as estimativas e análises dos custos e receitas: nessa etapa devem ser considerados todos os custos possíveis que a URRCD terá, desde gastos com energia elétrica, abastecimento de água, telefone e internet, gastos com folha de pagamento, manutenção de máquinas e equipamentos, gastos com financiamento, se houver. Ao projetar o FC, é preciso quantificar também a depreciação, a inflação e os impostos que serão pagos pela futura empresa, uma vez que eles podem afetar o desempenho do PI.

Para o cálculo da receita, deve-se tomar como base a população do município e a estimação da geração de RCD diária da cidade e da capacidade da URRCD. A primeira etapa é transformar a quantidade de RCD recebida em toneladas por hora e multiplicá-la pela capacidade de processamento da URRCD. Depois, essa quantidade processada é multiplicada pelo preço médio estimado de venda do agregado e multiplicada pelos dias trabalhados no ano. De posse da receita, pode-se partir para a projeção do FC para todo o horizonte de planejamento. Aqui devem ser considerados todos os dados referentes ao investimento inicial, aos custos fixos e variáveis e às receitas esperadas, previamente definidas.

Com o FC projetado, é preciso definir os critérios de retorno e riscos que serão incorporados ao plano de negócios: os indicadores de retorno e de riscos devem ser calculados nesse momento. O empreendedor pode optar pelo uso de somente alguns deles ou de todos, porém é importante frisar que o uso do maior número possível de indicadores leva à redução da incerteza quantos aos resultados obtidos no PI.

Na sequência, devem ser calculados os indicadores de viabilidade econômica: nessa etapa é efetivamente aplicada a MMIA via ferramenta computacional \$AVEPI, o qual é um aplicativo web livre desenvolvido pela Universidade Tecnológica Federal do Paraná - campus Pato Branco e está disponível no site da instituição (LIMA et al., 2017a). O \$AVEPI gera todos os indicadores de riscos, retorno, LEs e VLs, conforme é apresentado na Tabela 1.

Na etapa seguinte deve ser aplicada a SMC. A SMC busca simular os diversos componentes do FC em um modelo probabilístico e repetir o processo milhares de vezes (LIMA et al., 2017b). Dessa forma, pode-se obter a distribuição de probabilidades dos retornos de um PI (GITMAN; ZUTTER, 2017). Cada componente do FC segue distribuições de probabilidades específicas. As simulações foram feitas por meio do software Microsoft Excel ${ }^{\circledR}$ para as seguintes variáveis: preço

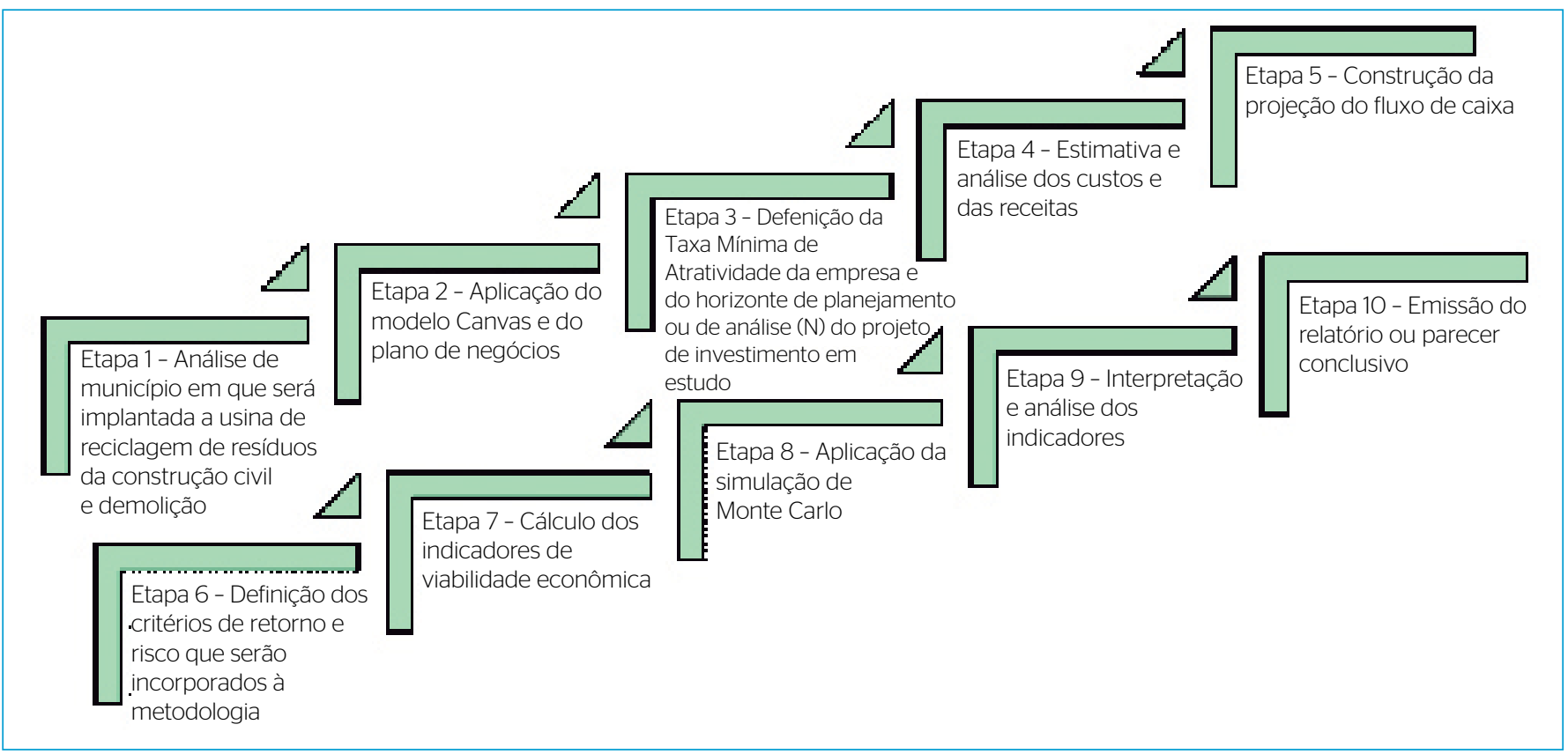

Figura 2 - Etapas do estudo de viabilidade econômico-financeira. 
de venda dos agregados, custo variável e fixo da usina, geração anual média de RCD e TMA. Na SMC, mediante geração de números aleatórios, valores são obtidos para essas variáveis, resultando em vários FCs e, consequentemente, em vários resultados para cada um dos indicadores considerados para o PI.

Pela repetição desse procedimento, por exemplo, 10 mil vezes, pode-se gerar a distribuição de frequências para cada indicador da MMIA, a qual permite aferir sua probabilidade de sucesso (LIMA et al., 2017b). Essa etapa transforma a demonstração de resultados e os FCs determinísticos em modelos estocásticos. De posse de todos os resultados gerados, devem-se fazer a interpretação e análise dos indicadores gerados por meio da MMIA via \$AVEPI e dos resultados obtidos da SMC, na qual se busca analisar a amplitude dos graus de riscos e retorno sobre o PI e quão variáveis são os LEs obtidos.

Por fim, faz-se a emissão do relatório e a elaboração do parecer conclusivo: a última etapa busca, unicamente, constatar se o PI é ou não viável econômica e financeiramente, isto é, faz-se a avaliação do mérito econômico do empreendimento. A Figura 3 apresenta um framework do modelo proposto neste trabalho para aplicação em municípios brasileiros. É uma figura-resumo de todas as interligações do modelo proposto para que o investidor possa realizar a avaliação do PI e verificar se ele apresenta a viabilidade esperada.

\section{Estudo de caso: aplicação no município de Pato Branco}

O município de Pato Branco está localizado na região sudoeste do estado do Paraná. Segundo dados do Instituto Brasileiro de Geografia e Estatística (IBGE, 2010), a população estimada do município é de 81.378 habitantes (município de médio porte), com projeção de crescimento de $1 \%$ ao ano até o ano de 2041 . O setor de construção civil no município está em um bom momento, com projeção de crescimento para os próximos anos. A geração média de RCD (kg/hab/dia) do município é de 0,569 , ou seja, cada habitante gera, aproximadamente, meio quilograma de resíduos da construção civil por dia (ABRELPE, 2016).

Com esses dados iniciais, seguindo o modelo proposto, parte-se para a montagem do modelo Canvas, o qual envolve nove blocos e é apresentado na Figura 4. Os blocos mostraram principalmente que os clientes da URRCD serão as próprias empresas de construção civil, as quais produzem os resíduos, bem como a prefeitura, que pode absorver a produção para utilizar nas principais obras do município. Os canais de comunicação da empresa serão, principalmente, por meio de contato direto com os potenciais clientes, mas também ocorrerá a divulgação em jornais de circulação local e propagandas em programas de rádio e televisão.

Entendeu-se que os recursos-chave da empresa envolvem matéria-prima de boa procedência, ou seja, o RCD recebido deve ser separado adequadamente, de forma que não prejudique o processo de reciclagem e o material reciclado. O espaço físico (terreno) no qual a URRCD será instalada também é um recurso-chave, pois, se bem localizada, reduz os custos de transporte tanto do RCD a ser reciclado para a usina quanto do agregado reciclado para os clientes. Uma boa equipe é essencial, e a gestão deve ser feita por profissional com experiência na área, da mesma forma que a operação das máquinas. As máquinas para o

Tabela 1 - Indicadores da metodologia multi-índice ampliada (MMIA)*.

\begin{tabular}{|c|c|c|c|}
\hline Dimensão & Indicador & Dimensão & Indicador \\
\hline \multirow{10}{*}{$\begin{array}{l}\text { Retorno } \\
\text { (SOUZA e CLEMENTE, 2009) }\end{array}$} & Valor Presente: VP & \multirow{7}{*}{$\begin{array}{c}\text { Limites de } \\
\text { Elasticidade } \\
\text { (LIMA et al., 2015) }\end{array}$} & $\Delta \% \mathrm{TMA}$ \\
\hline & Valor Presente Líquido: VPL & & $\Delta \% \mathrm{FC}_{0}$ \\
\hline & VP da Receita Total: VP(RT) & & $\Delta \% \mathrm{FC}_{\mathrm{j}}$ \\
\hline & VP do Custo Variável Total: VP(CVT) & & $\Delta \% \mathrm{FC}_{0}$ e FC \\
\hline & VP do Custo Fixo: VP(CF) & & $\Delta \% \mathrm{TMA}^{\mathrm{T}} \mathrm{FC}_{\mathrm{o}}$ \\
\hline & VP do Valor Residual: VP(VR) & & $\Delta \% \mathrm{TMA}^{\mathrm{T}} \mathrm{FC} \mathrm{j}_{\mathrm{j}}$ \\
\hline & Valor Presente Líquido Anualizado: VPLA & & $\Delta \% \mathrm{FC}_{0}$ e $\mathrm{FC}_{\mathrm{j}} \mathrm{e} \mathrm{TMA}$ \\
\hline & Índice Benefício Custo: IBC & \multirow{8}{*}{\multicolumn{2}{|c|}{$\begin{array}{l}\text { Legenda: } \Delta \% \text { = Variação percentual; } \\
\text { TMA = Taxa Mínima de Atratividade; } \\
\text { FC = Investimento inicial; } \\
\begin{aligned} \text { FC }_{\mathrm{j}}=\text { Fluxo de Caixa para o período j; } \\
\text { Q = Quantidade de vendas; } \\
\text { CF = Custo Fixo; } \\
\text { CT = Custo Total; } \\
\text { RT = Receita Total; e } \\
\text { VR = Valor Residual. }\end{aligned}\end{array}$}} \\
\hline & Retorno Adicional sobre o Investimento: ROIA & & \\
\hline & Índice ROIA/TMA & & \\
\hline \multirow{5}{*}{$\begin{array}{l}\text { Riscos } \\
\text { (SOUZA e CLEMENTE, 2009; } \\
\text { LIMA et al., 2013) }\end{array}$} & Payback ajustado & & \\
\hline & Taxa Interna de Retorno: TIR & & \\
\hline & Payback ajustado/N & & \\
\hline & Índice TMA/TIR & & \\
\hline & Payback descontado & & \\
\hline
\end{tabular}

*Todas as fórmulas para o cálculo dos indicadores da MMIA podem ser encontradas no site do \$AVEPI em <pb.utfpr.edu.br/savepi/>. Acesso em: 18 abr. 2016. Fonte: adaptado de Lima et al. (2015) e Gularte et al. (2017). 


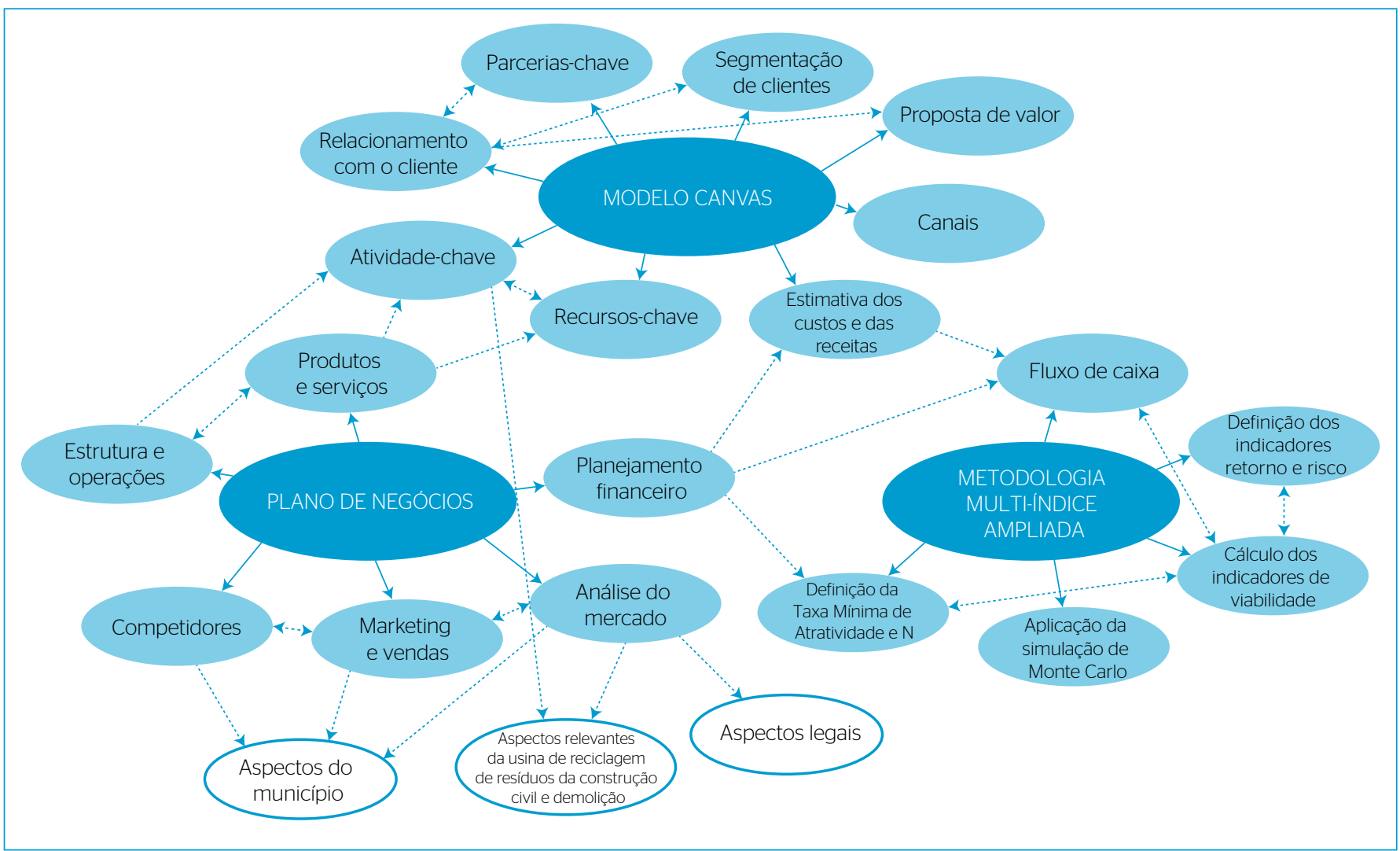

Figura 3 - Framework do modelo de avaliação proposto.

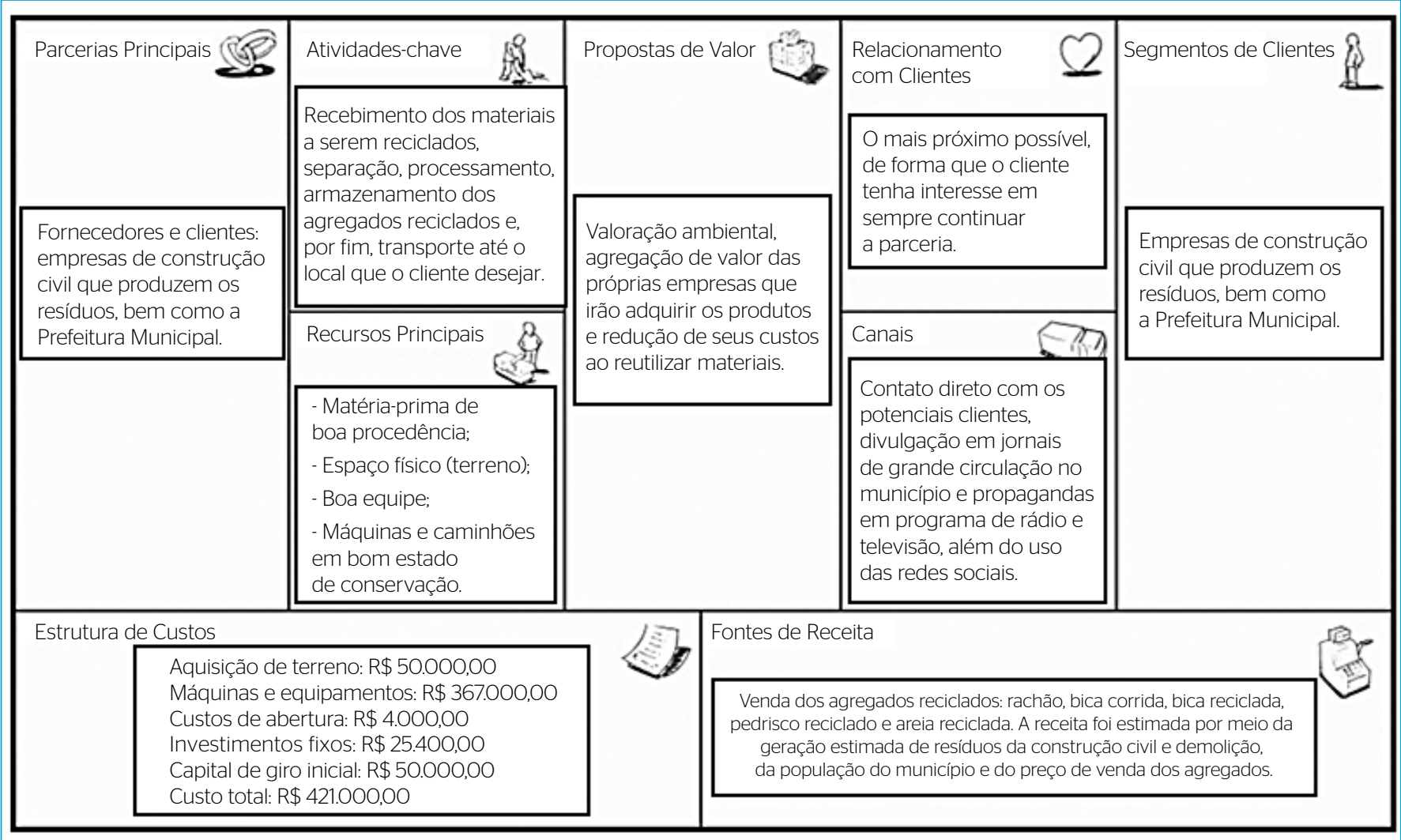

Figura 4 - Representação gráfica dos nove blocos do modelo Canvas. 
processamento do material precisam estar em bom estado de funcionamento, bem como os caminhões que fazem o transporte dos materiais.

O bloco do fluxo de receitas da URRCD - Pato Branco foi estimado com base na análise de outras usinas instaladas no Brasil, principalmente a URRCD de Maringá, na qual foi feita uma visita in loco das instalações. Verificou-se que, de todo o RCD recebido na usina, perde-se em torno de $20 \%$ ao fim do processo de reciclagem, o que está de acordo com os valores encontrados na literatura, vide Pinto (1999), principalmente em virtude da separação de materiais que não são recicláveis, como madeira e gesso. Para encontrar a receita anual, considerou-se, de acordo com a população estimada para o município em 2017, a média anual de RCD em toneladas. Para o cálculo do volume dos agregados reciclados foi considerada a menor taxa de conversão: 1,08 tonelada por metro cúbico, conforme Maqbrit (2016). Fazendo essa conversão e multiplicando pelo preço de venda do agregado reciclado, foi encontrada a receita prévia; por fim, descontam-se os $20 \%$ de materiais não recicláveis, e essa é a receita total anual. É importante destacar também que a produção de RCD do município depende do tamanho da população deste, assim, pela estimativa de crescimento da população feita pelo IBGE para os 25 anos do PI, foram previstas as quantidades diárias geradas de RCD. O preço de venda dos agregados foi atualizado de acordo com a previsão oficial do IBGE para a inflação nos próximos anos (4,5\% ao ano).

As principais atividades da empresa envolvem o recebimento dos materiais a serem reciclados, a separação destes, o envio às máquinas para processamento, o transporte para o local a ser armazenado e, por fim, o transporte até o local que o cliente desejar. Outra atividade importante a ser desenvolvida é a de relacionamento com o cliente. O gestor, principalmente, precisa estabelecer um bom relacionamento com os clientes, de forma que eles tenham interesse em continuar adquirindo agregados reciclados da URRCD para suas obras.

O bloco dos principais parceiros da empresa também serão, basicamente, os clientes dela, daí a importância de um bom relacionamento com eles. Em pesquisa realizada na cidade de Pato Branco, encontrou-se como maiores construtoras (em faturamento anual) e potenciais clientes: Construtora Pato Branco, Construtora Centauro, All Green Construtora, Construtora Palanger, TNP Construtora e Incorporadora, Construtora Ouro Verde, VP Construções, Construtora Amadeu Pereira e Tonial Construções,

Por fim, o bloco 9, que traz a estrutura de custos da empresa, foi estimado por meio de pesquisa de mercado na cidade e região. As despesas pré-operacionais foram estimadas em $\mathrm{R} \$ 421 \mathrm{mil}$, os quais incluem a aquisição do terreno, da usina propriamente dita e de outros equipamentos - pá, caçamba, balança - necessários para seu funcionamento. Os investimentos fixos totalizaram $\mathrm{R} \$ 25.400$, e o capital de giro $\mathrm{R} \$ 50 \mathrm{mil}$. As despesas com pessoal foram estimadas em $\mathrm{R} \$ 106.306,68$, e o pró-labore $\mathrm{R} \$ 72$ mil por ano. Os custos fixos e variáveis anuais foram estimados em R\$ 242.400, incluindo água, luz, telefone, manutenção, diesel, fretes etc.
No total, os custos pré-operacionais somados às despesas com pessoal e aos custos fixos e variáveis totalizaram $\mathrm{R} \$ 917.106,70$. Em virtude da limitação de páginas, não foi possível detalhar toda a estrutura de custos da empresa. Para mais informações sobre como a estrutura foi montada e calculada, ver Gularte (2017).

Após serem previstos todos os custos e as receitas, pode ser projetado o FC para todo o período do investimento (25 anos). Os valores do FC do empreendimento foram estimados para um horizonte de 25 anos, considerando as receitas e os custos no período em que ocorrerão, de acordo com a inflação estimada (Índice de Preços ao Consumidor Amplo de 4,5\% ao ano) e com o aumento da população prevista pelo IBGE (2010). Este trabalho considerou que todos os elementos do FC são afetados da mesma forma pela inflação, ou seja, foi considerado um cenário de inflação homogênea.

A empresa enquadra-se no Simples Nacional, programa do governo que permite consolidar os impostos da empresa em uma taxa de acordo com a receita bruta da empresa. Além disso, a empresa optará por financiar o PI. Do montante a ser investido, 75\% pode ser financiado pela Caixa Econômica Federal, cuja taxa de juro é de $9 \%$ ao ano. A amortização pode ser feita em até 15 anos, com carência de cinco anos. Para este estudo, considerou-se o sistema de amortização constante. Assim, a amortização da dívida, bem como os juros a serem pagos, foram considerados no FC. A depreciação também foi considerada e, conforme a Instrução Normativa nº 162/98 da Receita Federal do Brasil, o prazo de depreciação de máquinas da construção civil é de quatro anos com uma taxa de $25 \%$ ao ano, resultando, assim, no valor de R 61.750 por ano. O valor residual foi estimado em R\$ 30 mil.

Com a finalização de todos os blocos do modelo Canvas, deve-se construir o plano de negócios, o qual é a principal ferramenta de gestão do empreendedor, auxiliando-o a tomar decisões (DORNELAS et al., 2016). O plano de negócios envolve várias etapas, e a primeira é a de análise de mercado e competidores. Conforme explicitado anteriormente, há a tendência de que as empresas, preocupadas com suas imagens perante os clientes, invistam na sustentabilidade, principalmente por meio da reciclagem e reutilização dos materiais que antes seriam descartados em lixões ou aterros. Quanto às perspectivas de mercado, ainda há de se destacar o crescimento da população, que leva a uma demanda crescente por habitação. No que se refere aos competidores, não há, na região, nenhuma URRCD instalada hoje, portanto, ao menos no período inicial de atividades, não haveria competidores.

A análise seguinte do plano de negócios refere-se aos produtos e serviços oferecidos pela URRCD. Verificou-se que os principais agregados a serem vendidos seriam o pedrisco reciclado, a areia reciclada, o rachão e as bicas corrida e reciclada. Isso ocorre em virtude de serem os materiais que mais seriam utilizados em outras obras, pois servem de base para as suas partes estruturais. Além desses, verificou-se a possibilidade de, no futuro, iniciar a produção na própria URRCD de alguns subprodutos dos agregados reciclados, como tijolos, meio-fio e tubos para esgoto. 
A seção seguinte do plano de negócios é a de marketing e vendas. Como a URRCD fará o beneficiamento de materiais descartados em obras e reformas, é de suma importância que o empreendedor busque apresentar os benefícios do produto em comparação aos já existentes no mercado, tanto em relação aos custos da obra quanto em relação ao impacto socioambiental positivo. Portanto, é necessário que o empreendedor tenha um ótimo relacionamento com seus clientes, mantendo sempre contato para reforçar os benefícios de se usar agregados reciclados nas obras. Outro aspecto que auxilia nas vendas é o preço. Conforme pesquisa in loco na URRCD de Maringá, o preço de venda dos agregados reciclados deve ser em torno de $35 \%$ do valor dos produtos quando comprados novos.

Após terminar o plano de negócios, o passo seguinte consiste em calcular os indicadores determinísticos de retorno e riscos com base na MMIA via \$AVEPI, por meio do FC estimado. Destaca-se que os indicadores de RG e RN não foram contemplados neste estudo, pois dependem de análises ambientais mais profundas (HARZER, 2015). Os resultados obtidos são apresentados na Tabela 2. Optou-se por calcular todos os indicadores de retorno e riscos apresentados na metodologia, por possibilitar visão mais ampla dos resultados econômicos esperados para PI em estudo.

Os indicadores de retorno mostram que o VPL, o qual indica a riqueza proporcionada pelo PI (em toda a sua vida útil), corresponde

Tabela 2 - Indicadores da Metodologia Multi-índice Ampliada (MMIA).

\begin{tabular}{|c|c|c|c|}
\hline \multicolumn{2}{|l|}{ Retorno } & \multicolumn{2}{|c|}{ Limites de Elasticidade } \\
\hline Indicador & Resultado & Indicador & Resultado \\
\hline VP & $R \$ 704.856,49$ & $\Delta \% \mathrm{TMA}$ & $182,12 \%$ \\
\hline VPL & $\mathrm{R} \$ 580.756,49$ & $\Delta \% \mathrm{FC}_{0}$ & $117,66 \%$ \\
\hline $\mathrm{VP}(\mathrm{RT})$ & $\mathrm{R} \$$ 6.019.387,80 & $\Delta \% \mathrm{FC}_{j}$ & $54,06 \%$ \\
\hline VP (CVT) & $\mathrm{R} \$ 1.805 .285,26$ & $\Delta \% \mathrm{FC}_{0}$ e FC & $37,04 \%$ \\
\hline VP (CF) & $\mathrm{R} \$ 799.483,47$ & $\Delta \%$ TMA e FC & $71,45 \%$ \\
\hline $\mathrm{VP}(\mathrm{CT})$ & $\mathrm{R} \$ 2.604 .768,74$ & $\Delta \%$ TMA e FC & $41,66 \%$ \\
\hline VP (VR) & $\mathrm{R} \$ 1.669 .02$ & $\Delta \% \mathrm{FC}_{0}$ e $F C_{j}$ e TMA & 20,38\% \\
\hline VPLA & $\mathrm{R} \$ 75.333,80$ & & \\
\hline $\mathrm{IBC}$ & 2,1766 & & \\
\hline ROIA & $3,16 \%$ & & \\
\hline Índice ROIA/TMA & $25,79 \%$ & & \\
\hline \multicolumn{4}{|l|}{ Riscos } \\
\hline Indicador & Resultado & & \\
\hline Payback ajustado & 12 anos & & \\
\hline $\mathrm{TIR}$ & $34,56 \%$ & & \\
\hline Paybackajustado/N & $48,00 \%$ & & \\
\hline Índice TMA/TIR & $35,44 \%$ & & \\
\hline
\end{tabular}

$\Delta \%$ : variação percentual; TMA: taxa mínima de atratividade; $\mathrm{FC}_{0}$ : investimento inicial; FC: fluxo de caixa para o período j; Q: quantidade de vendas; PVu: preço de venda unitário; CVu: custo variável unitário; $\mathrm{MCu}$ : margem de contribuição unitária; CF: custo fixo; CT: custo total; RT: receita total; VR: valor residual. ao valor esperado de aproximadamente $\mathrm{R} \$ 580.756,49$, o equivalente a R $\$ 75.333,80$ por período (VPLA). Nesse PI, a cada unidade monetária investida, estima-se o retorno de 2,17 unidades, valor observado para o IBC. Isso implica um ROIA de $3,16 \%$ ao período, além da taxa mínima de atratividade de $12,25 \%$ ao ano, isto é, $25,79 \%$ acima do ganho oportunizado pelo mercado se for aplicado a essa TMA medido pelo índice ROIA/TMA. De acordo com esse ganho, o PI pode ser classificado na categoria de retorno de grau médio-alto, seguindo a classificação proposta por Lima et al. (2017a).

Os indicadores de riscos mostram que, na relação entre o tempo de retorno do capital investido (payback) e a vida útil considerada $(\mathrm{N})$, medida pelo índice payback/N, o PI apresenta risco de $48 \%$, indicando um grau médio (LIMA et al., 2017a) de o projeto não se pagar (SOUZA; CLEMENTE, 2009). Ao medir a proximidade entre a TMA e a TIR, utilizando o índice TMA/TIR, o PI também apresentou um indicativo de grau de risco classificado como baixo-médio (35,44\%). Quanto ao indicador payback, como foi explicado na metodologia, aqui se optou por utilizar o payback ajustado (LIMA et al., 2013), o qual objetiva corrigir possíveis distorções que possam ser geradas no cálculo do payback em projetos que envolvam financiamento externo à organização proponente. Dessa forma, o índice payback ajustado mostrou que o PI em estudo levará 12 anos para a recuperação do capital investido.

No tocante aos LEs, o PI em estudo torna-se inviável economicamente se a TMA aumentar mais do que $182,12 \%$ ou se o investimento inicial $\left(\mathrm{FC}_{0}\right)$ realizado aumentar mais do que $117,66 \%$ do valor projetado, desde que mantidos constantes os demais parâmetros. Já a variação entre o investimento inicial, os demais fluxos de caixa e a TMA não pode ser maior que 54,06\% para que o PI continue sendo viável.

A etapa seguinte foi transformar as projeções dos FCs determinísticos em modelos estocásticos com o uso da SMC. Para a composição do modelo estocástico foi utilizada a distribuição triangular, com variabilidade de $20 \%$ para mais e para menos por meio dos dados históricos, seguindo orientação de especialistas da área consultados. A SMC foi elaborada abrangendo 10 mil interações entre as variáveis aleatórias. Com base nessas simulações, geraram-se os novos FC para os 25 períodos e, com eles, foram estimados os novos valores para os indicadores das dimensões riscos, retorno e sensibilidades.

O VPL médio ficou em $\mathrm{R} \$ 583.627,12$, próximo ao valor observado na abordagem determinística. O IBC apresentou retorno muito próximo ao determinístico, com 2,17 unidades de retorno para cada unidade monetária investida. Na média, o índice ROIA/TMA estabeleceu-se em $27,30 \%$, sendo considerado um risco de grau baixo-médio (LIMA et al., 2017a). No que diz respeito às probabilidades do VPL, verificou-se que, com 10 mil simulações, a probabilidade de o VPL ser menor que zero é de apenas $0,22 \%$. Por outro lado, a probabilidade do PI ser menor que R\$ 1 milhão é de 94,05\%, e a probabilidade de ele ser maior que R\$ 500 mil é de $62,29 \%$. 


\section{CONSIDERAÇÕES FINAIS}

O setor da construção civil é expressivo para o crescimento do país. Esse setor é responsável pela contratação de parte da mão de obra, além de ter contribuição relevante na formação do produto interno bruto do país. Porém, na contramão desses benefícios, ele é um grande gerador de resíduos, os quais geralmente são destinados incorretamente. Dessa forma, este artigo teve como objetivo desenvolver um modelo que permita uma avaliação de oportunidade de implantação de URRCD em municípios brasileiros, bem como aplicá-lo, por meio de um estudo de caso, na cidade de Pato Branco.

Os resultados encontrados satisfazem aos objetivos propostos neste estudo. No aspecto financeiro, a implantação da URRCD mostrou-se economicamente viável. Com os resultados gerados pela MMIA via \$AVEPI, verificou-se que todos os indicadores foram positivos. Os indicadores da dimensão retorno mostraram que o PI apresenta boa perspectiva de retorno, com destaque para o VPLA, o qual mostrou rendimento anual satisfatório para o investidor, e o indicador ROIA/TMA, que apresentou retorno de 25,79\%. Além disso, os indicadores da dimensão riscos também mostraram-se favoráveis. Utilizando o indicador payback ajustado, espera-se que o retorno do capital investido ocorra em 12 anos. Os índices payback/N e TMA/TIR ficaram em 48 e 35,44\%, respectivamente, sendo categorizados como índices de grau de riscos baixo-médio (LIMA et al., 2017a).

No tocante aos LEs, verificou-se que o PI em estudo é mais sensível às reduções nos FCs. Os indicadores de sensibilidades, considerando a escala proposta por Lima et al. (2017a), mostram que a TMA deveria variar na ordem de $182,12 \%$ para que o mercado financeiro propiciasse o mesmo retorno que o projeto, ou seja, a sensibilidade mostra-se de grau excepcional, mesmo grau apresentado pela $\Delta \%$ custos de implantação $\left(\mathrm{FC}_{0}\right)$, de $117,66 \%$. A $\Delta \% \mathrm{TMA}_{\text {e }} \mathrm{FC}_{0}(71,45 \%)$ apresentou grau médio. A $\Delta \% \mathrm{FC}_{\mathrm{j}} \mathrm{de}$ $54,06 \%$ e a $\triangle \%$ TMA e FCj $(41,66 \%)$ mostraram-se sensíveis de grau médio. Por fim, a $\Delta \%$ entre $\mathrm{FC}_{0}$ e $\mathrm{FC}_{\mathrm{j}}$ de $37,04 \%$ mostrou-se de grau baixo-médio. Diante disso, a análise preliminar mostra que a decisão de investir no PI em estudo apresenta-se como uma boa opção de investimento.

Entretanto, em razão da aleatoriedade dos componentes do FC projetado, recorreu-se à SMC como forma de minimizar o risco envolvido no PI. Por meio dessa técnica, verificou-se que o VPL médio ficou próximo do calculado na abordagem determinística (MMIA via \$AVEPI), bem como o VPLA, o IBC, o ROIA e o ROIA/TMA. Já os índices de riscos resultaram em valores menores na abordagem estocástica, ou seja, há risco menor envolvido no projeto do que o estimado pela abordagem determinística, mostrando que essa abordagem pode ser mais confiável. Isso corrobora com os resultados observados por Harzer (2015), segundo o qual o índice TMA/TIR tende a superestimar o risco de um PI.

Portanto, a análise determinística apresentou resultados favoráveis à decisão de investir no PI, e a análise estocástica apresentou resultados similares, mostrando que há alta probabilidade (99,78\%) de que o VPL do PI na URRCD de Pato Branco seja positivo. Entretanto, o payback ajustado mostrou que se espera retorno do investimento após 12 anos de operação da URRCD. Uma forma de melhorar esse resultado seria via incentivo do poder público, por meio de subsídio à aquisição do terreno e dos equipamentos de instalação. Com isso, os custos de instalação da URRCD diminuiriam, levando a um aumento da atratividade financeira do projeto. Os índices de elasticidade mostraram que é preciso monitorar as receitas esperadas e os custos estimados com mais atenção. Assim, será preciso um trabalho, por parte dos gestores da URRCD, em prol de sempre estarem buscando aumentar a demanda pelos agregados reciclados, como maneira de aumentar a receita da usina, desde que ela não ultrapasse sua capacidade de produção.

Como sugestão para estudos futuros, propõe-se que, além do modelo proposto, seja proposta uma análise do valor ambiental da URRCD, como forma de qualificar os resultados em relação aos clientes que comprarão os agregados reciclados para usar em suas obras. Essa análise também agregará valor ao empreendimento perante a sociedade ao mostrar todos os benefícios proporcionados pela gestão adequada dos resíduos provenientes de construção civil e demolição. Sugere-se também que sejam feitas a avaliação da viabilidade de receber RCD de municípios próximos à sede da URRCD, bem como a avaliação de projetos de união de vários municípios para montagem da URRCD. Por fim, sugere-se a ampliação do modelo proposto, por meio da incorporação de tópicos da TOR.

\section{REFERÊNCIAS}

ASSOCIAÇÃO BRASILEIRA DE EMPRESAS DE LIMPEZA PÚBLICA E RESÍDUOS ESPECIAIS (ABRELPE). (2016) Panorama nos resíduos sólidos no Brasil: 2016. São Paulo: ABRELPE.

ASSOCIAÇÃO BRASILEIRA DE NORMAS TÉCNICAS (ABNT). (2004) NBR 15114: Resíduos sólidos da construção civil - Áreas de reciclagem - Diretrizes para projeto, implantação e operação. Rio de Janeiro: ABNT.
ASSOCIAÇÃO BRASILEIRA PARA RECICLAGEM DE RESÍDUOS DA CONSTRUÇÃO CIVIL E DEMOLIÇÃO (ABRECON). (2O15) Relatório: pesquisa setorial 2014/2015. São Paulo: ABRECON.

BRASIL. (1998) Instrução Normativa SRF no 162, de 31 de dezembro de 1998. Fixa prazo de vida útil etaxa de depreciação dos bens que relaciona. Disponível em: http://normas.receita.fazenda.gov.br/sijut2consulta/link.ac tion?visao=anotado\&idAto=15004. Acesso em: 30 nov. 2015. 
BRASIL. (2002) Ministério do Meio Ambiente. Conselho Nacional do Meio Ambiente (CONAMA). Resolução no 307, de 5 de julho de 2002. Diário Oficial da União, no 136, p. 95-96. Disponível em: <http://www.mma.gov.br>. Acesso em: 15 ago. 2015.

CASAROTTOFILHO, N.KOPITTKE,B.H.(2010)Análise de/nvestimentos: Matemática Financeira, Engenharia Econômica, Tomada de Decisão, Estratégia Empresarial. 11. ed. São Paulo: Atlas. 411 p.

COELHO, A.;BRITO, J.(2O13) Economic viability analysis of a construction and demolition waste recycling plant in Portugal-part I: location, materials, technology and economic analysis. Journal of Cleaner Production, v. 39, p. 338-352. https://doi.org/10.1016/j.jclepro.2012.08.024

CORREIA NETO, J.F. (2009) Elaboração e avaliação de projetos de investimento: considerando o risco. Rio de Janeiro: Elsevier.

DORNELAS, J.; BIM, A.; FREITAS, G.; USHIKUBO, R. (2016) Plano de negócios com o modelo Canvas: guia prático de avaliação de ideias de negócio a partir de exemplos. Rio de Janeiro: LTC.

DURAN, X.; LENIHAN, H.; O'REGAN, B. (2006) A model for assessing the economic viability of construction and demolition waste recycling and the case of Ireland. Resources, Conservation and Recycling, v. 46, n. 3, p. 302-320. https://doi.org/10.1016/j. resconrec.2005.08.003

GAVA, E.M. (2014) Concepção e análise de modelos de negócios por meio do Business Model Canvas. Monografia (MBA em Gestão Empresarial) - Universidade do Extremo Sul Catarinense, Criciúma.

GITMAN, L.J.; ZUTTER, C.J. (2017) Princípios de administração financeira. 14. ed. São Paulo: Pearson Education do Brasil.

GUJARATI, D.N. (2002) Econometria básica. 3. ed. São Paulo: Makron Books.

GULARTE, L.C.P. (2017) Modelo de avaliação da viabilidade econômico-financeira da implantação de usinas de reciclagem de resíduos da construção civil em municípios brasileiros. Dissertação (Mestrado em Engenharia de Produção) - Universidade Tecnológica Federal do Paraná, Pato Branco.

GULARTE, L.C.P.; LIMA, J.D.; OLIVEIRA, G.A.; TRENTIN, M.G.; SETTI, D. Estudo de viabilidade econômica da implantação de uma usina de reciclagem de resíduos da construção civil no município de Pato Branco - PR utilizando a metodologia multi-índice ampliada. Engenharia Sanitária e Ambiental, v. 22, n. 5, p. 985-992, 2017. https:// doi.org/10.1590/s1413-41522017162097

HARZER, J.H. (2015) Indicadores de riscos em projetos de investimentos: uma contribuição à Metodologia Multi-indice. Tese (Doutorado em Administração) - Pontifícia Universidade Católica do Paraná, Curitiba.

INSTITUTO BRASILEIRO DE GEOGRAFIA E ESTATÍSTICA (IBGE). (2010) Censo Demográfico de 2010. Disponível em: <http://www. ibge.gov.br/home/>. Acesso em: 20 set. 2016.
LIMA, J.D. de; BENNMANN, M.; SOUTHIER, L.F.P.; BATISTUS, D.R.; OLIVEIRA, G.A. (2017a) \$AV€P - Web System to Support the Teaching and Learning Process in Engineering Economics. Brazilian Journal of Operations and Production Management, v. 14, n. 4, p. 469-485.

LIMA, J.D. de; SCHEITT, L.C.; BOSCHI, T.F.; SILVA, N.J.; MEIRA, A.A.; DIAS, G.H. (2013) Propostas de ajuste no cálculo do payback de projetos de investimentos financiados. Custos e @gronegócio on line, v. 9, n. 4. p. 162-180

LIMA, J.D. de; TRENTIN, M.G.; OLIVEIRA, G.A.; BATISTUS, D.R.; SETTI, D. (2015) A systematic approach for the analysis of the economic viability of investment projects. International Journal of Engineering Management and Economics, v. 5, n. 1/2, p. 19-34. https://doi.org/10.1504/IJEME.2015.069887

LIMA, J.D. de; TRENTIN, M.G.; OLIVEIRA, G.A.; BATISTUS, D.R.; SETTI, D. (2017b) Systematic Analysis of Economic Viability with Stochastic Approach: A Proposal for Investment. In: AMORIM, M.; FERREIRA, C.; VIEIRA JUNIOR, M.; PRADO, C. (orgs.). Engineering Systems and Networks. Suíça: Springer International. p. 317-325. https://doi.org/10.1007/978-3-319-45748-2_34

MAQBRIT. Disponível em: <http://www.maqbrit.com.br/britagem. html>. Acesso em: 12 abr. 2016.

NOGAS, P.S.M.; SOUZA, A.; SILVA, W.V. (2011) Análise de investimentos: uma contribuição probabilística ao índice TMA/TIR da Metodologia Multi-índice. Revista Iberoamericana de Ciencias Empresariales y Economía, v. 2, n. 2, p. 43-55.

NUNES, K.R.A.; MAHLER, C.F.; VALLE, R.; NEVES, C. (2007) Evaluation of investments in recycling centers for construction and demolition wastes in Brazilian municipalities. Waste Management, v. 27, n. 11, p. 1531-1540. https://doi.org/10.1016/j.wasman.2006.09.007

PINTO, T.P. (1999) Metodologia para a gestão diferenciada de Resíduos sólidos da construção urbana. Tese (Doutorado) - Escola Politécnica da Universidade de São Paulo. Departamento de Engenharia de Construção Civil, São Paulo.

SANTOS, F.R.; POMPEU, R.B. (2014) Logística reversa de resíduos da construção civil: análise de viabilidade econômica. Revista Tecnológica Fatec Americana, v. 2, n. 1, p. 105-120.

SOUZA, A.; CLEMENTE, A. (2009) Decisões Financeiras e Análise de Investimentos: fundamentos, técnicas e aplicações. São Paulo: Atlas.

ZHAO, W.; LEEFTINK, R.B.; ROTTER, V.S. (2010) Evaluation of the economic feasibility for the recycling of construction and demolition waste in China: the case of Chongqing. Resources, Conservation and Recycling, v. 54, n. 6, p. 377-389. https://doi. org/10.1016/j.resconrec.2009.09.003

\$AVEPI. Sistema de Análise da Viabilidade Econômica de Projetos de Investimento. Disponível em: <http://pb.utfpr.edu.br/savepi/ creditos.php>. Acesso em: fev. 2018. 